\title{
Current results of open total arch replacement versus hybrid thoracic endovascular aortic repair for aortic arch aneurysm: A meta-analysis of comparative studies
}

\author{
Umberto Benedetto, MD, PhD, ${ }^{a}$ Giovanni Melina, $\mathrm{MD}, \mathrm{PhD},{ }^{\mathrm{a}}$ Emiliano Angeloni, $\mathrm{MD},{ }^{\mathrm{a}}$ \\ Massimiliano Codispoti, MD, FRCS, ${ }^{\mathrm{b}}$ and Riccardo Sinatra, MD, ${ }^{\mathrm{a}}$ Rome, Italy, and Cambridge, UK
}

For aortic arch aneurysm, conventional open total aortic arch replacement (OTAAR) has long been considered the standard therapy. Despite improvement of surgical technology and strategy, however, OTAAR remains a procedure associated with some morbidity and mortality, particularly among high-risk patients. ${ }^{1}$

In an attempt to reduce the complications associated with OTAAR, hybrid thoracic endovascular aortic repair (HTEAR), with aortic arch debranching end endovascular graft placement, has emerged as an attractive option for high-risk patients. ${ }^{2}$ Randomized controlled trial comparing the strategies are not available, however, and potential advantages of the hybrid strategy relative to the conventional procedure remain to be demonstrated. To gain insights into the role of the hybrid approach in the management of aortic arch aneurysm, we conducted a meta-analysis of available comparative studies.

\footnotetext{
From the Department of Cardiac Surgery, ${ }^{\text {a }}$ Ospedale S. Andrea, La Sapienza, Università di Roma, Rome, Italy; and Department of Cardiac Surgery, ${ }^{\mathrm{b}}$ Papworth Hospital NHS Foundation Trust, Cambridge, UK.

Disclosures: Authors have nothing to disclose with regard to commercial support. Presented at Aortic Symposium 2012, New York, NY, April 26-27, 2012.

Received for publication July 20, 2012; revisions received Aug 19, 2012; accepted for publication Sept 12, 2012; available ahead of print Oct 5, 2012.

Address for reprints: Umberto Benedetto, MD, PhD, Department of Cardiac Surgery,

La Sapienza, Università di Roma, Ospedale Sant'Andrea, Via di Grottarossa 1035,

00189 Rome, Italy (E-mail: u2benedetto@libero.it).

J Thorac Cardiovasc Surg 2013;145:305-6

$0022-5223 / \$ 36.00$

Copyright (C) 2013 by The American Association for Thoracic Surgery

http://dx.doi.org/10.1016/j.jtcvs.2012.09.011
}

\section{MATERIALS AND METHODS}

All studies comparing the results of hybrid (HTEAR) and open (OTAAR) approaches for the repair of aortic arch aneurysm were identified by means of a 2-level search strategy. First, a public-domain database (MEDLINE) was searched by using a Web-based search engine (PubMed, Embase, Cochrane Library). Second, relevant studies were identified through a manual search of secondary sources, including references of initially identified articles and a search of reviews and commentaries. The MEDLINE database was searched from January 1966 through June 2012. Medical subjects heading key words included "aortic arch aneurysm," "total open aortic arch replacement/repair," and "hybrid endovascular aortic arch repair."

Studies considered for inclusion met the following criteria: results of OTAAR and HTEAR for aortic arch aneurysm were compared, and at least 1 of the outcomes of interest was reported. Outcomes investigated were operative mortality, permanent neurologic deficit, late mortality, and need for reintervention. For each study, data regarding outcomes of interest were used to generate an odds ratio ( $<1$ favoring HTEAR; $>1$ favoring OTAAR) and a rate ratio $(<1$ favoring HTEAR; $>1$ favoring OTAAR). The $95 \%$ confidence intervals (CIs) were based on the asymptotic normality of the combined estimates. To account for undetectable heterogeneity related to the observational design of the studies included, pooled estimates were calculated by means of a random effect model. Between-study heterogeneity was analyzed by means of $I^{2}$, and a value $>50 \%$ was considered to be indicative of heterogeneity. Publication bias was evaluated by means of the Begg-Mazumdar rank correlation test.

\section{RESULTS}

Our research identified 4 comparative observational studies $^{2-5}$ that included a total of 378 patients (OTAAR, $\mathrm{n}=269$; HTEAR, $\mathrm{n}=109$ ). All studies reported data on operative mortality and permanent neurologic deficit, all but 1 study $^{3}$ reported data on late mortality, and 2 studies ${ }^{3,4}$ reported on need for reintervention. Among studies

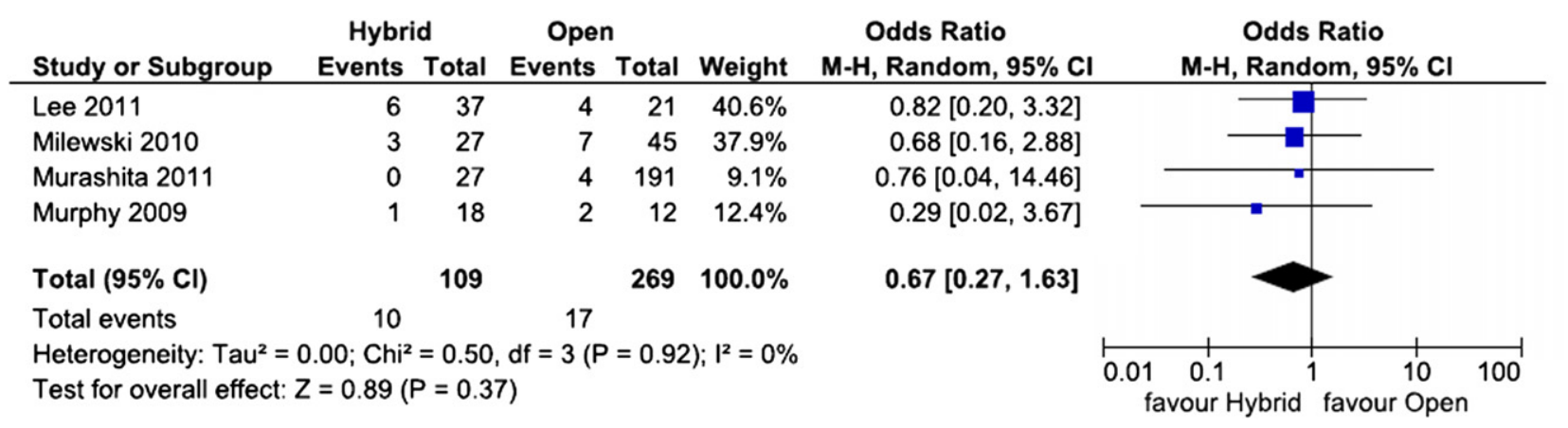

FIGURE 1. Meta-analysis for operative mortality of hybrid versus open approach. The odds ratios for death from each included study (squares) and from the pooled estimate (diamond) are plotted, each with $95 \%$ confidence interval ( $C I$; line lengths and width of diamond). $M-H$, Mantel-Haenszel test. 


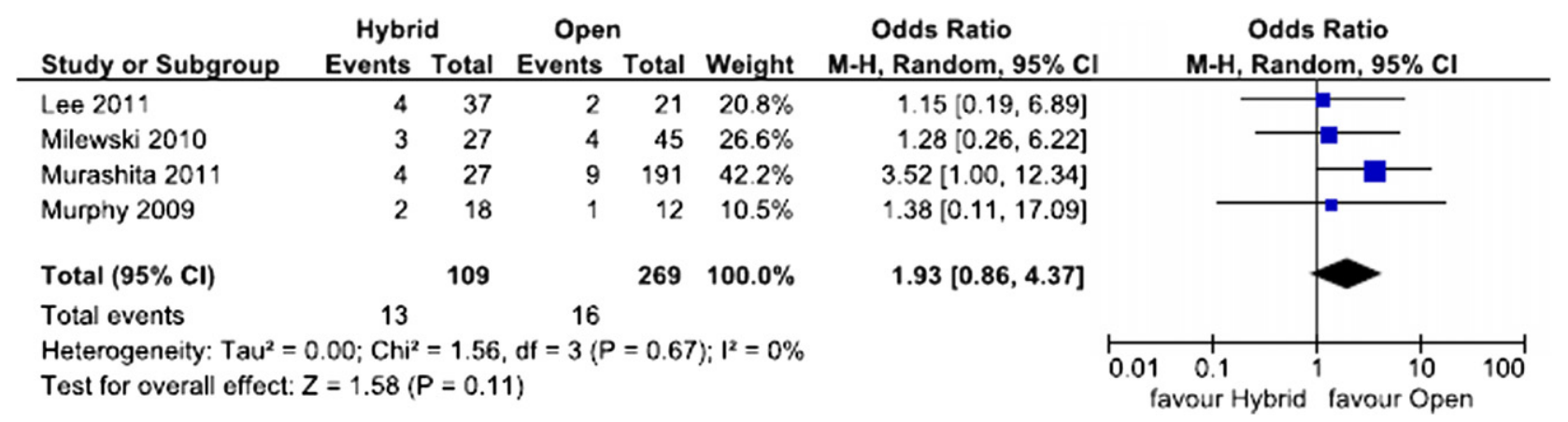

FIGURE 2. Meta-analysis for permanent neurologic deficit associated with hybrid versus open approach. The odds ratios for neurologic deficit from each included study (squares) and from the pooled estimate (diamond) are plotted, each with $95 \%$ confidence interval ( $C I$; line lengths and width of diamond). $M-H$, Mantel-Haenszel test.

reporting on late outcomes, follow-up ranged from 7 to 18.5 months.

Pooled analysis of operative outcomes showed that HTEAR did not significantly improve operative mortality (odds ratio, 0.67; 95\% CI, 0.27-1.63; $P=.92$; Figure 1 ), whereas it was associated with a slight, nonsignificant increase in permanent neurologic deficits (odds ratio, 1.93 ; 95\% CI, 0.86-4.37; $P=.1$; Figure 2). A nonsignificant trend toward increased late mortality was observed after HTEAR (rate ratio, 1.73; 95\% CI, 0.9-3.3; $P=.10$ ), without any differences with regard to need for reintervention (rate ratio, 0.81; 95\% CI, 0.32-2.07; $P=.66)$. No heterogeneity was found among outcomes of interest $\left(I^{2}=0 \%\right.$ for all tests), and no publication bias was detected $(P=.69)$.

\section{DISCUSSION}

Surgical complexity and frailty of patients make aortic arch aneurysms a challenging surgical indication. ${ }^{1}$ Several studies have shown combined aortic debranching followed by endovascular repair to be an effective and feasible means of treating aortic arch aneurysms ${ }^{2-5}$; however, definitive conclusions regarding its superiority to the conventional open approach are still lacking.

This meta-analysis, which pooled data from available comparative reports on HTEAR versus OTAAR, showed no significant improvement with respect to operative and late mortality with HTEAR. Surprisingly, HTEAR was actually found to be associated with a slight increase in the incidence of permanent neurologic deficit, suggesting that the risk of embolism remains significant even with
HTEAR. As a consequence, careful manipulation and a short crossclamp time during debranching should be considered mandatory.

The main limitation of this meta-analysis was the observational design of the studies included, which makes the analysis prone to biases caused by, for example, selection of participants, different patient risk profiles, and unavailability for follow-up. When potential heterogeneity among studies was investigated, however, it was found to be nonsignificant for all outcomes of interest.

In conclusion, no definitive evidence supports the superiority of the HTEAR relative to OTAAR. Surgical strategy for aortic arch aneurysm should be chosen on the basis of the patient's characteristics, and OTAAR should be still considered the benchmark for alternative approach evaluation.

\section{References}

1. Minatoya K, Ogino H, Matsuda H, Sasaki H, Tanaka H, Kobayashi J, et al. Is conventional aortic arch surgery justifiable in octogenarians? J Thorac Cardiovasc Surg. 2010;139:641-5.

2. Milewski RK, Szeto WY, Pochettino A, Moser GW, Moeller P, Bavaria JE. Have hybrid procedures replaced open aortic reconstruction in high-risk patients? A comparative study of elective open arch debranching with endovascular stent graft placement and conventional elective open total and distal aortic arch reconstruction. J Thorac Cardiovasc Surg. 2010;140:590-7.

3. Murphy EH, Beck AW, Clagett P, DiMaio JM, Jessen ME, Arko FR. Combined aortic debranching and thoracic endovascular aneurysm repair (TEVAR), effective but at a cost. Arch Surg. 2009;144:222-7.

4. Lee CW, Beaver TM, Klodell CT Jr, Hess PJ Jr, Martin TD, Feezor RJ, et al. Arch debranching versus elephant trunk procedures for hybrid repair of thoracic aortic pathologies. Ann Thorac Surg. 2001;91:465-71.

5. Murashita T, Matsuda H, Domae K, Iba Y, Tanaka H, Sasaki H, et al. Less invasive surgical treatment for aortic arch aneurysms in high-risk patients: a comparative study of hybrid thoracic endovascular aortic repair and conventional total arch replacement. J Thorac Cardiovasc Surg. 2012;143:1007-13. 